\title{
Prediction of Lighweight Concrete Panel Homogeneity by Ultrasonic Pulse Velocity (UPV)
}

\author{
Agus Santoso, Darmono, Slamet Widodo, Faqih Ma'arif \\ Universitas Negeri Yogyakarta \\ Yogyakarta, Indonesia \\ agussan@uny.ac.id
}

\begin{abstract}
Lightweight concrete is defined as concrete having a specific gravity ranging from $600-1600 \mathrm{~kg} / \mathrm{m}^{3}$, the specific gravity of this concrete is lower than normal concrete. In this paper, the homogeneity of lightweight concrete panel wall is evaluated using the non-destructive - ultrasonic pulse velocity method. This study involved about 12 different composition panel wall and core tested in 28 days of age, the direct method was used on 180 monitoring points of specimens. The Influence of several factors on the experiment results were evaluated, including expanded polystyrene, polypropylene fibers, SCM thickness, and core wall panel thickness. Comparison between ultrasonic pulse velocity and empirical test showed that the ultrasonic pulse velocity is very useful to evaluate homogeneity and quality of composite materials.
\end{abstract}

Keywords-UPV; homogeneity; wall panel; direct method

\section{INTRODUCTION}

Lightweight concrete is a concrete that has a density lighter than the concrete in general. In general, light weight concrete weighs between $600-1600 \mathrm{~kg} / \mathrm{m}^{3}$ (SNI 2847-2002). Basically lightweight concrete is obtained by the addition of aggregates that have mild characteristics into the mixture, thus the use of lightweight concrete can significantly reduce the weight of the structure.

The use of lightweight concrete as the main materials using stryrofoam waste, has been studied by Agus, et al. [1] which is used as a wall of building structures. In this study, styrofoam is used because it is in fact an environmentally friendly material, including: (1) not releasing toxic and hazardous substances, without chlorofluorocarbon (CFC) or hydro chlorofluorocarbon (HCFC), inhibiting mold growth, having mechanical and thermal characteristics for buildings; (2) does not cause allergic effects and does not harm the health of those who produce and the workers; (3) Recyclable: any styrofoam waste is cut into granules thus it does not cause waste of production.

The combination of styrofoam waste as the core layer, polypropylene fiber as a coating is one of the products that has been produced in this study. In the application, the material can be made as a wall panel and is one of the composite structures. Until now, the problems that arise related to composite system is the condition of the interface layer between elements in the core layer and the coating which is expected to work collaboratively bearing the external load. The next problem with the existence of polypropylene fiber which is one of the additive materials that can bias the wave [2] but has a tensile strength and very good resistance to the shock load, so this type of material is applied in the manufacture of composite wall panels called self-compacting mortar.

The key word in the success of making composite system is material homogeneity. This study focuses more on the homogeneity of a composite material in which the use of Styrofoam material is more dominant to form the core layer on the wall panel. Analysis of material homogeneity can be conducted by using non-destructive testing namely Ultrasonic Pulse Velocity (UPV). UPV testing has been widely practiced in developed countries and has grown rapidly with various methods and media processed using modern technology. Research conducted by Benaicha, et al. [3], Benaicha, et al. [4], Bogas, Al-Mufti and Fried [5], Petro and Kim [6], Hamidian, et al. [7] and Lorenzi, et al. [8] have proven the reliability of this method in evaluating the performance of a concrete structure and material.

However, based on the review on previous studies, there are not specific studies have been found that specifically discuss the homogeneity of mixed composite panel systems, which is composed of wave forming properties combined with porous materials (which can be easily passed by waves). Specific investigation is conducted and also to simultaneously assess the reliability of UPV tools in evaluating various core thicknesses and self-compacting mortar layers, as well as the proportion of polypropylene fibers to be used. Meanwhile, the amount of polypropylene fiber used refers to Agus, et al. [1] and Slamet [9] which states that the use of optimum fiber is 1 $\mathrm{kg} / \mathrm{m}^{3}$.

Ultrasonic pulse velocity testing use the direct method, because it has the best reading accuracy [10], as for direct testing in this study serves to determine the amount of material homogeneity, the influence of fiber usage, the thickness of selfcompacting mortar layer, and the thickness of the layer core. Statistical analysis was performed as a comparison of experimental test results, as well as proving the excellent accuracy of reading in direct method. 


\section{EXPERIMENTAL PROGRAM}

\section{A. Materials and Mix Proportion}

Materials used in this study were fine aggregate of Progo, Kulonprogo, Yogyakarta; Portland pozzolan cement (PPC) type I from Gresik Indonesia. The mix design consisted of of self-compacting mortar (SCM) 1Pc:4Ps $+1.2 \%$ of Sikament $\mathrm{NN}+$ polypropylene fibers $\left(0 \mathrm{~kg} / \mathrm{m}^{3}\right.$ and $\left.1.0 \mathrm{~kg} / \mathrm{m}^{3}\right)$; the type of fiber is polypropylene monofilament with $18 \mu \mathrm{m}$ in diameter and $12 \mathrm{~mm}$ in length, coupling agent, styrofoam waste, and wiremesh.

TABLE 1. MIX PROPORTION OF CORE PANEL

\begin{tabular}{|c|l|c|}
\hline No & \multicolumn{1}{|c|}{ Materials } & $\begin{array}{c}\text { Materials Requirement Per Cubic Meter } \\
\left(\mathbf{k g} / \mathbf{m}^{\mathbf{3}}\right)\end{array}$ \\
\hline 1 & Styrofoam & 14.3 \\
\hline 2 & PPC & 350 \\
\hline 3 & Fine aggregate & 435.7 \\
\hline 4 & Water & 200 \\
\hline
\end{tabular}

TABLE 2. MIX PROPORTION of SCM

\begin{tabular}{|c|l|c|}
\hline \multirow{2}{*}{ No. } & \multicolumn{1}{|c|}{ Materials } & $\begin{array}{c}\text { Materials Requirement Per Cubic } \\
\text { Meter }(\mathbf{k g})\end{array}$ \\
\hline 1 & Fine aggregate & 381.4 \\
\hline 2 & PPC & 1525.4 \\
\hline 3 & Water & 343.2 \\
\hline 4 & Sikament NN & 3.88 \\
\hline \multirow{2}{*}{5} & $\begin{array}{l}\text { Volume fraction of PP } \\
\text { fibers }\end{array}$ & 0.0 \\
\cline { 3 - 3 } & & 1.0 \\
\hline
\end{tabular}

TABLE 3. MIX PROPORTION of 12 PANEL

\begin{tabular}{|c|l|c|c|c|c|c|}
\hline No & Specimen & $\begin{array}{c}\text { Core } \\
(\mathbf{m m})\end{array}$ & $\begin{array}{c}\text { SCM } \\
(\mathbf{m m})\end{array}$ & $\begin{array}{c}\text { PPF } \\
(\mathbf{k g} / \mathbf{m 3})\end{array}$ & $\begin{array}{c}\text { Slump } \\
\mathbf{f l o w} \\
(\mathbf{m m})\end{array}$ & $\begin{array}{c}\text { Thickness } \\
(\mathbf{m m})\end{array}$ \\
\hline 1 & PL 90 $\mathrm{Ra}$ & 90 & 15 & 0 & 630 & 119.5 \\
\hline 2 & PL $90_{0} \mathrm{Rb}$ & 90 & 15 & 0 & 630 & 118.8 \\
\hline 3 & PL $90_{1} \mathrm{Fa}$ & 90 & 15 & 1 & 526 & 120.5 \\
\hline 4 & PL $90_{1} \mathrm{Fb}$ & 90 & 15 & 1 & 526 & 121.3 \\
\hline 5 & PL $80_{0} \mathrm{Ra}$ & 80 & 20 & 0 & 630 & 118.3 \\
\hline 6 & PL $80_{0} \mathrm{Rb}$ & 80 & 20 & 0 & 630 & 121.5 \\
\hline 7 & PL $80_{1} \mathrm{Fa}$ & 80 & 20 & 1 & 526 & 122.6 \\
\hline 8 & PL $80_{1} \mathrm{Fb}$ & 80 & 20 & 1 & 526 & 118.8 \\
\hline 9 & PL 70 $\mathrm{Ra}$ & 70 & 25 & 0 & 630 & 119.7 \\
\hline 10 & PL $70_{0} \mathrm{Rb}$ & 70 & 25 & 0 & 630 & 121.0 \\
\hline 11 & PL 70 $\mathrm{Fa}$ & 70 & 25 & 1 & 526 & 120.6 \\
\hline 12 & PL $70_{1} \mathrm{Fb}$ & 70 & 25 & 1 & 526 & 120.3 \\
\hline
\end{tabular}

\section{B. Ultrasonic Pulse Velocity Methods}

According to European standards EN 12504-4, BS EN 12504-4 and ASTM C597-02 (2003), 2 transmission mode were used. This system is transmitted with a sender frequency of $54 \mathrm{kHz}$, in accordance with the industry standards. This nominal frequency serves to limit the depth of the ultrasonic wave emission and the minimum thickness of the specimen to be tested. With the distance traveled by wave is "L" and the propagation time is " $t$ " in the material, the wave reaches the second transducer thus, and it is possible to calculate the speed of wave propagation in a material: $\mathrm{V}=\mathrm{L} / \mathrm{T}$.

Measurements are conducted by the direct method, by placing the transducer and receiver face to face with minimum thickness distance of $100 \mathrm{~mm}$, UPV procedure scheme is presented in Fig. 1 and Fig. 2. UPV testing results can be used to check homogeneity and control the quality of concrete [11] [12] [13] [14].



Fig. 1. Schematic of UPV Apparatus (Source: ASTM C597-02 2003)

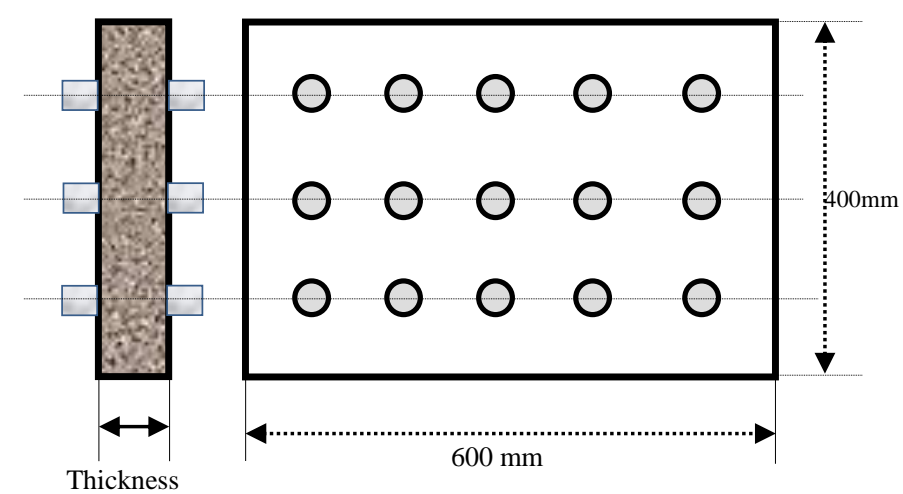

Fig. 2. Direct method on Wall Panel

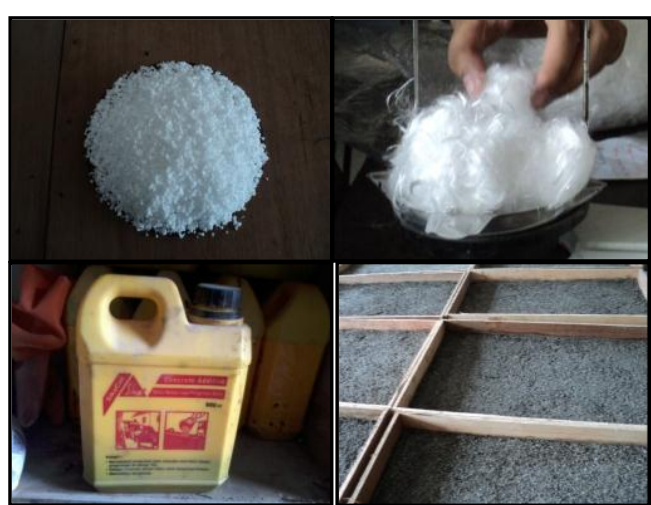

Fig 3. Material used in experimen

Note:

(1) Expanded polystyrene; (2) Polypropylene fibers; (3) Sikament NN; (4) Core panel

\section{TEST RESULTS AND DISCUSSION}

Ultrasonic pulse velocity testing is conducted by the direct method. The tests were performed on the thickness of the core layers and the thickness of different SCM layers, the total wall thickness was $120 \mathrm{~mm}$. 
A. Ultrasonic Pulse Velocity Measurement

The ultrasonic wave propagation speed results in the form of travel time $(\Delta t)$, which is then analyzed to produce a speed value. The magnitude of wave propagation speed is presented in Table 4.

TABLE 4. DIRECT TRANSMISSION

\begin{tabular}{|c|c|c|c|c|c|}
\hline No & Specimen & $\begin{array}{l}\text { Meas. } \\
\text { points }\end{array}$ & $\begin{array}{c}\text { Transducer } \\
(\mathbf{m m})\end{array}$ & $\begin{array}{l}\text { TT } \\
(\Delta t)\end{array}$ & $\begin{array}{c}\mathbf{V} \\
(\mathbf{m} / \mathbf{s})\end{array}$ \\
\hline \multirow{15}{*}{1} & \multirow{15}{*}{ PL 70 $0 R_{a}$} & $\mathrm{~A}_{1}$ & 118.30 & 84.50 & 1.40 \\
\hline & & $\mathrm{A}_{2}$ & 119.40 & 66.33 & 1.80 \\
\hline & & $\mathrm{A}_{3}$ & 122.00 & 71.76 & 1.70 \\
\hline & & $\mathrm{A}_{4}$ & 119.00 & 74.38 & 1.60 \\
\hline & & $\mathrm{A}_{5}$ & 119.10 & 79.40 & 1.50 \\
\hline & & $\mathrm{A}_{6}$ & 119.00 & 74.38 & 1.60 \\
\hline & & $\mathrm{A}_{7}$ & 119.60 & 66.44 & 1.80 \\
\hline & & $\mathrm{A}_{8}$ & 121.40 & 71.41 & 1.70 \\
\hline & & $\mathrm{A}_{9}$ & 119.00 & 74.38 & 1.60 \\
\hline & & $\mathrm{A}_{10}$ & 119.00 & 74.38 & 1.60 \\
\hline & & $\mathrm{A}_{11}$ & 118.00 & 65.56 & 1.80 \\
\hline & & $\mathrm{A}_{12}$ & 120.00 & 66.67 & 1.80 \\
\hline & & $\mathrm{A}_{13}$ & 121.00 & 75.63 & 1.60 \\
\hline & & $\mathrm{A}_{14}$ & 118.30 & 69.59 & 1.70 \\
\hline & & $\mathrm{A}_{15}$ & 119.10 & 79.40 & 1.50 \\
\hline \multirow{15}{*}{2} & \multirow{15}{*}{ PL $70_{0} R_{b}$} & $\mathrm{~A}_{1}$ & 123.80 & 73.69 & 1.68 \\
\hline & & $\mathrm{A}_{2}$ & 121.40 & 61.62 & 1.97 \\
\hline & & $\mathrm{A}_{3}$ & 120.30 & 87.17 & 1.38 \\
\hline & & $\mathrm{A}_{4}$ & 119.50 & 78.62 & 1.52 \\
\hline & & $\mathrm{A}_{5}$ & 117.70 & 80.62 & 1.46 \\
\hline & & $\mathrm{A}_{6}$ & 121.00 & 75.16 & 1.61 \\
\hline & & $\mathrm{A}_{7}$ & 121.40 & 78.83 & 1.54 \\
\hline & & $\mathrm{A}_{8}$ & 120.90 & 77.01 & 1.57 \\
\hline & & $\mathrm{A}_{9}$ & 119.40 & 75.57 & 1.58 \\
\hline & & $\mathrm{A}_{10}$ & 118.30 & 74.87 & 1.58 \\
\hline & & $\mathrm{A}_{11}$ & 116.30 & 76.01 & 1.53 \\
\hline & & $\mathrm{A}_{12}$ & 116.80 & 73.92 & 1.58 \\
\hline & & $\mathrm{A}_{13}$ & 116.00 & 74.36 & 1.56 \\
\hline & & $\mathrm{A}_{14}$ & 111.30 & 79.50 & 1.40 \\
\hline & & $\mathrm{A}_{15}$ & 118.00 & 84.89 & 1.39 \\
\hline \multirow{15}{*}{3} & \multirow{15}{*}{$\mathrm{PL} 70_{1} \mathrm{~F}_{\mathrm{a}}$} & $\mathrm{A}_{1}$ & 121.00 & 69.14 & 1.75 \\
\hline & & $\mathrm{A}_{2}$ & 122.00 & 79.74 & 1.53 \\
\hline & & $\mathrm{A}_{3}$ & 120.80 & 76.46 & 1.58 \\
\hline & & $\mathrm{A}_{4}$ & 120.00 & 63.49 & 1.89 \\
\hline & & $\mathrm{A}_{5}$ & 120.50 & 74.84 & 1.61 \\
\hline & & $\mathrm{A}_{6}$ & 121.00 & 67.98 & 1.78 \\
\hline & & $\mathrm{A}_{7}$ & 120.50 & 74.84 & 1.61 \\
\hline & & $\mathrm{A}_{8}$ & 120.60 & 68.91 & 1.75 \\
\hline & & $\mathrm{A}_{9}$ & 119.80 & 65.46 & 1.83 \\
\hline & & $\mathrm{A}_{10}$ & 120.50 & 79.28 & 1.52 \\
\hline & & $\mathrm{A}_{11}$ & 120.10 & 70.23 & 1.71 \\
\hline & & $\mathrm{A}_{12}$ & 120.00 & 75.95 & 1.58 \\
\hline & & $\mathrm{A}_{13}$ & 121.00 & 75.16 & 1.61 \\
\hline & & $\mathrm{A}_{14}$ & 119.00 & 73.91 & 1.61 \\
\hline & & $\mathrm{A}_{15}$ & 120.20 & 79.60 & 1.51 \\
\hline \multirow{13}{*}{4} & \multirow{13}{*}{ PL 70 ${ }_{1} F_{b}$} & $\mathrm{~A}_{1}$ & 120.50 & 55.53 & 2.17 \\
\hline & & $\mathrm{A}_{2}$ & 121.00 & 55.76 & 2.17 \\
\hline & & $\mathrm{A}_{3}$ & 122.50 & 53.96 & 2.27 \\
\hline & & $\mathrm{A}_{4}$ & 122.20 & 56.31 & 2.17 \\
\hline & & $\mathrm{A}_{5}$ & 122.00 & 57.82 & 2.11 \\
\hline & & $\mathrm{A}_{6}$ & 120.00 & 54.30 & 2.21 \\
\hline & & $\mathrm{A}_{7}$ & 121.00 & 52.61 & 2.30 \\
\hline & & $\mathrm{A}_{8}$ & 122.00 & 58.37 & 2.09 \\
\hline & & $\mathrm{A}_{9}$ & 121.50 & 55.99 & 2.17 \\
\hline & & $\mathrm{A}_{10}$ & 121.10 & 61.16 & 1.98 \\
\hline & & $\mathrm{A}_{11}$ & 119.00 & 61.98 & 1.92 \\
\hline & & $\mathrm{A}_{12}$ & 121.00 & 55.00 & 2.20 \\
\hline & & $\mathrm{A}_{13}$ & 122.30 & 54.60 & 2.24 \\
\hline
\end{tabular}

\begin{tabular}{|c|c|c|c|c|c|}
\hline No & Specimen & $\begin{array}{l}\text { Meas. } \\
\text { points }\end{array}$ & $\begin{array}{c}\text { Transducer } \\
\text { (mm) }\end{array}$ & $\begin{array}{l}\text { TT } \\
(\Delta t)\end{array}$ & $\begin{array}{c}\mathbf{V} \\
(\mathbf{m} / \mathbf{s})\end{array}$ \\
\hline & & $\mathrm{A}_{14}$ & 122.20 & 55.55 & 2.20 \\
\hline & & $\mathrm{A}_{15}$ & 121.70 & 57.68 & 2.11 \\
\hline \multirow{15}{*}{5} & \multirow{15}{*}{$\mathrm{PL} 80_{0} \mathrm{R}_{\mathrm{a}}$} & $\mathrm{A}_{1}$ & 116.60 & 57.44 & 2.03 \\
\hline & & $\mathrm{A}_{2}$ & 116.90 & 64.59 & 1.81 \\
\hline & & $\mathrm{A}_{3}$ & 116.80 & 65.99 & 1.77 \\
\hline & & $\mathrm{A}_{4}$ & 116.80 & 63.48 & 1.84 \\
\hline & & $\mathrm{A}_{5}$ & 118.10 & 62.16 & 1.90 \\
\hline & & $\mathrm{A}_{6}$ & 118.00 & 60.51 & 1.95 \\
\hline & & $\mathrm{A}_{7}$ & 118.30 & 60.05 & 1.97 \\
\hline & & $\mathrm{A}_{8}$ & 119.70 & 62.34 & 1.92 \\
\hline & & $\mathrm{A}_{9}$ & 120.30 & 62.33 & 1.93 \\
\hline & & $\mathrm{A}_{10}$ & 120.20 & 63.26 & 1.90 \\
\hline & & $\mathrm{A}_{11}$ & 118.40 & 57.20 & 2.07 \\
\hline & & $\mathrm{A}_{12}$ & 119.40 & 62.84 & 1.90 \\
\hline & & $\mathrm{A}_{13}$ & 118.50 & 62.37 & 1.90 \\
\hline & & $\mathrm{A}_{14}$ & 118.90 & 55.56 & 2.14 \\
\hline & & $\mathrm{A}_{15}$ & 117.60 & 62.22 & 1.89 \\
\hline \multirow{15}{*}{6} & \multirow{15}{*}{ PL $80_{0} R_{b}$} & $\mathrm{~A}_{1}$ & 123.70 & 54.02 & 2.29 \\
\hline & & $\mathrm{A}_{2}$ & 120.40 & 50.38 & 2.39 \\
\hline & & $\mathrm{A}_{3}$ & 121.40 & 55.94 & 2.17 \\
\hline & & $\mathrm{A}_{4}$ & 121.90 & 58.61 & 2.08 \\
\hline & & $\mathrm{A}_{5}$ & 120.50 & 62.76 & 1.92 \\
\hline & & $\mathrm{A}_{6}$ & 124.00 & 55.61 & 2.23 \\
\hline & & $\mathrm{A}_{7}$ & 120.50 & 54.52 & 2.21 \\
\hline & & $\mathrm{A}_{8}$ & 123.00 & 54.19 & 2.27 \\
\hline & & $\mathrm{A}_{9}$ & 122.00 & 56.22 & 2.17 \\
\hline & & $\mathrm{A}_{10}$ & 119.50 & 64.25 & 1.86 \\
\hline & & $\mathrm{A}_{11}$ & 122.20 & 63.65 & 1.92 \\
\hline & & $\mathrm{A}_{12}$ & 120.30 & 58.97 & 2.04 \\
\hline & & $\mathrm{A}_{13}$ & 121.60 & 61.73 & 1.97 \\
\hline & & $\mathrm{A}_{14}$ & 121.40 & 60.40 & 2.01 \\
\hline & & $\mathrm{A}_{15}$ & 120.00 & 58.54 & 2.05 \\
\hline \multirow{15}{*}{7} & \multirow{15}{*}{$\mathrm{PL} 80_{1} \mathrm{~F}_{\mathrm{a}}$} & $\mathrm{A}_{1}$ & 124.20 & 70.97 & 1.75 \\
\hline & & $\mathrm{A}_{2}$ & 120.90 & 63.63 & 1.90 \\
\hline & & $\mathrm{A}_{3}$ & 122.40 & 70.75 & 1.73 \\
\hline & & $\mathrm{A}_{4}$ & 122.10 & 66.00 & 1.85 \\
\hline & & $\mathrm{A}_{5}$ & 124.40 & 72.75 & 1.71 \\
\hline & & $\mathrm{A}_{6}$ & 123.50 & 69.38 & 1.78 \\
\hline & & $\mathrm{A}_{7}$ & 121.30 & 68.15 & 1.78 \\
\hline & & $\mathrm{A}_{8}$ & 122.70 & 61.04 & 2.01 \\
\hline & & $\mathrm{A}_{9}$ & 122.00 & 67.78 & 1.80 \\
\hline & & $\mathrm{A}_{10}$ & 124.70 & 69.28 & 1.80 \\
\hline & & $\mathrm{A}_{11}$ & 123.10 & 67.27 & 1.83 \\
\hline & & $A_{12}$ & 121.30 & 67.77 & 1.79 \\
\hline & & $\mathrm{A}_{13}$ & 122.10 & 64.60 & 1.89 \\
\hline & & $\mathrm{A}_{14}$ & 121.60 & 61.41 & 1.98 \\
\hline & & $\mathrm{A}_{15}$ & 122.70 & 66.68 & 1.84 \\
\hline \multirow{15}{*}{8} & \multirow{15}{*}{$\mathrm{PL} 80_{1} \mathrm{~F}_{\mathrm{b}}$} & $\mathrm{A}_{1}$ & 117,60 & 54.19 & 2.17 \\
\hline & & $\mathrm{A}_{2}$ & 118,30 & 54.52 & 2.17 \\
\hline & & $\mathrm{A}_{3}$ & 119,40 & 52.60 & 2.27 \\
\hline & & $\mathrm{A}_{4}$ & 121,10 & 55.81 & 2.17 \\
\hline & & $\mathrm{A}_{5}$ & 111,20 & 52.70 & 2.11 \\
\hline & & $\mathrm{A}_{6}$ & 118,60 & 53.67 & 2.21 \\
\hline & & $\mathrm{A}_{7}$ & 117,70 & 51.17 & 2.30 \\
\hline & & $\mathrm{A}_{8}$ & 119.90 & 57.37 & 2.09 \\
\hline & & $\mathrm{A}_{9}$ & 120.40 & 55.48 & 2.17 \\
\hline & & $\mathrm{A}_{10}$ & 121.30 & 61.26 & 1.98 \\
\hline & & $\mathrm{A}_{11}$ & 117.20 & 61.04 & 1.92 \\
\hline & & $\mathrm{A}_{12}$ & 118.20 & 53.73 & 2.20 \\
\hline & & $\mathrm{A}_{13}$ & 120.40 & 53.75 & 2.24 \\
\hline & & $\mathrm{A}_{14}$ & 118.50 & 53.86 & 2.20 \\
\hline & & $\mathrm{A}_{15}$ & 121.90 & 57.77 & 2.11 \\
\hline \multirow{4}{*}{9} & \multirow{4}{*}{ PL $90_{0} R_{a}$} & $A_{1}$ & 120.00 & 68.57 & 1.75 \\
\hline & & $\mathrm{A}_{2}$ & 120.20 & 62.93 & 1.91 \\
\hline & & $\mathrm{A}_{3}$ & 120.00 & 63.83 & 1.88 \\
\hline & & $\mathrm{A}_{4}$ & 119.00 & 64.32 & 1.85 \\
\hline
\end{tabular}




\begin{tabular}{|c|c|c|c|c|c|}
\hline No & Specimen & $\begin{array}{l}\text { Meas. } \\
\text { points }\end{array}$ & $\begin{array}{c}\text { Transducer } \\
(\mathbf{m m})\end{array}$ & $\begin{array}{l}\text { TT } \\
(\Delta \mathbf{t})\end{array}$ & $\begin{array}{c}\mathbf{V} \\
(\mathbf{m} / \mathbf{s}) \\
\end{array}$ \\
\hline & & $\mathrm{A}_{5}$ & 119.20 & 68.11 & 1.75 \\
\hline & & $\mathrm{A}_{6}$ & 119.00 & 65.38 & 1.82 \\
\hline & & $\mathrm{A}_{7}$ & 121.20 & 66.96 & 1.81 \\
\hline & & $\mathrm{A}_{8}$ & 120.00 & 67.04 & 1.79 \\
\hline & & $\mathrm{A}_{9}$ & 118.20 & 61.88 & 1.91 \\
\hline & & $\mathrm{A}_{10}$ & 120.40 & 66.15 & 1.82 \\
\hline & & $\mathrm{A}_{11}$ & 121.00 & 66.48 & 1.82 \\
\hline & & $\mathrm{A}_{12}$ & 120.20 & 65.68 & 1.83 \\
\hline & & $\mathrm{A}_{13}$ & 119.20 & 68.51 & 1.74 \\
\hline & & $\mathrm{A}_{14}$ & 118.00 & 66.29 & 1.78 \\
\hline & & $\mathrm{A}_{15}$ & 118.20 & 65.67 & 1.80 \\
\hline \multirow{15}{*}{10} & \multirow{15}{*}{ PL $90_{0} R_{b}$} & $\mathrm{~A}_{1}$ & 123.00 & 54.19 & 2.27 \\
\hline & & $\mathrm{A}_{2}$ & 122.40 & 57.20 & 2.14 \\
\hline & & $\mathrm{A}_{3}$ & 120.00 & 48.00 & 2.50 \\
\hline & & $\mathrm{A}_{4}$ & 121.00 & 53.30 & 2.27 \\
\hline & & $\mathrm{A}_{5}$ & 121.60 & 56.30 & 2.16 \\
\hline & & $\mathrm{A}_{6}$ & 122.80 & 60.79 & 2.02 \\
\hline & & $\mathrm{A}_{7}$ & 122.20 & 56.84 & 2.15 \\
\hline & & $\mathrm{A}_{8}$ & 120.00 & 49.79 & 2.41 \\
\hline & & $\mathrm{A}_{9}$ & 120.80 & 51.85 & 2.33 \\
\hline & & $\mathrm{A}_{10}$ & 122.40 & 55.38 & 2.21 \\
\hline & & $\mathrm{A}_{11}$ & 120.00 & 55.30 & 2.17 \\
\hline & & $\mathrm{A}_{12}$ & 120.00 & 52.63 & 2.28 \\
\hline & & $\mathrm{A}_{13}$ & 119.00 & 51.74 & 2.30 \\
\hline & & $\mathrm{A}_{14}$ & 120.00 & 55.05 & 2.18 \\
\hline & & $\mathrm{A}_{15}$ & 120.00 & 59.41 & 2.02 \\
\hline \multirow{15}{*}{11} & \multirow{15}{*}{$\mathrm{PL} 90_{1} \mathrm{~F}_{\mathrm{a}}$} & $\mathrm{A}_{1}$ & 120.00 & 96.77 & 1.24 \\
\hline & & $\mathrm{A}_{2}$ & 121.00 & 94.53 & 1.28 \\
\hline & & $\mathrm{A}_{3}$ & 120.00 & 91.60 & 1.31 \\
\hline & & $\mathrm{A}_{4}$ & 121.00 & 94.53 & 1.28 \\
\hline & & $\mathrm{A}_{5}$ & 120.00 & 90.91 & 1.32 \\
\hline & & $\mathrm{A}_{6}$ & 120.40 & 93.33 & 1.29 \\
\hline & & $\mathrm{A}_{7}$ & 121.00 & 98.37 & 1.23 \\
\hline & & $\mathrm{A}_{8}$ & 120.80 & 95.87 & 1.26 \\
\hline & & $\mathrm{A}_{9}$ & 121.00 & 91.67 & 1.32 \\
\hline & & $\mathrm{A}_{10}$ & 120.00 & 93.75 & 1.28 \\
\hline & & $\mathrm{A}_{11}$ & 120.00 & 96.77 & 1.24 \\
\hline & & $\mathrm{A}_{12}$ & 122.00 & 91.04 & 1.34 \\
\hline & & $\mathrm{A}_{13}$ & 121.80 & 95.16 & 1.28 \\
\hline & & $\mathrm{A}_{14}$ & 120.00 & 93.02 & 1.29 \\
\hline & & $\mathrm{A}_{15}$ & 119.60 & 91.30 & 1.31 \\
\hline \multirow{15}{*}{12} & \multirow{15}{*}{ PL $90{ }_{1} \mathrm{~F}_{\mathrm{b}}$} & $\mathrm{A}_{1}$ & 119.80 & 78.30 & 1.53 \\
\hline & & $\mathrm{A}_{2}$ & 120.00 & 65.22 & 1.84 \\
\hline & & $\mathrm{A}_{3}$ & 120.00 & 69.36 & 1.73 \\
\hline & & $\mathrm{A}_{4}$ & 120.00 & 82.76 & 1.45 \\
\hline & & $\mathrm{A}_{5}$ & 121.00 & 91.67 & 1.32 \\
\hline & & $\mathrm{A}_{6}$ & 120.50 & 93.41 & 1.29 \\
\hline & & $\mathrm{A}_{7}$ & 122.00 & 89.71 & 1.36 \\
\hline & & $\mathrm{A}_{8}$ & 121.70 & 83.36 & 1.46 \\
\hline & & $\mathrm{A}_{9}$ & 121.00 & 87.68 & 1.38 \\
\hline & & $\mathrm{A}_{10}$ & 121.50 & 61.68 & 1.97 \\
\hline & & $\mathrm{A}_{11}$ & 119.80 & 97.40 & 1.23 \\
\hline & & $\mathrm{A}_{12}$ & 118.50 & 84.04 & 1.41 \\
\hline & & $\mathrm{A}_{13}$ & 118.70 & 95.73 & 1.24 \\
\hline & & $\mathrm{A}_{14}$ & 119.00 & 92.97 & 1.28 \\
\hline & & $\mathrm{A}_{15}$ & 121.30 & 97.82 & 1.24 \\
\hline
\end{tabular}

Note:

PL $80_{0} \mathrm{Ra}$ : Panel, $80 \mathrm{~mm}$ core thickness ( $0 \%$ PPF), Reference a (specimen a) PL $80_{1} \mathrm{Fa}$ : Panel with $80 \mathrm{~mm}$ core thickness (1\%PPF), Fa (specimen a)

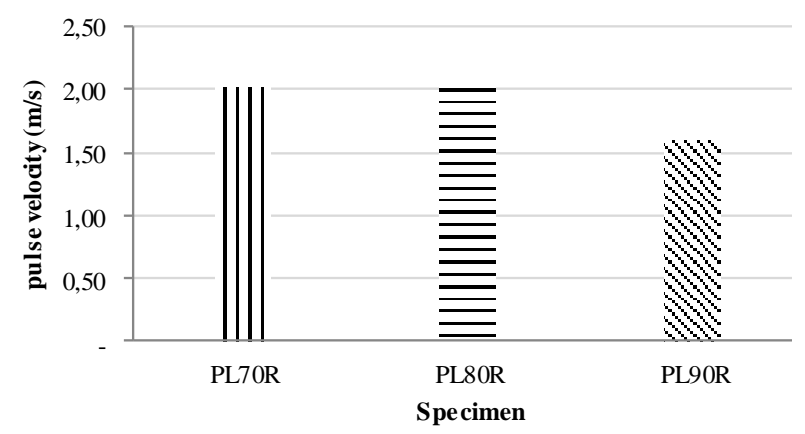

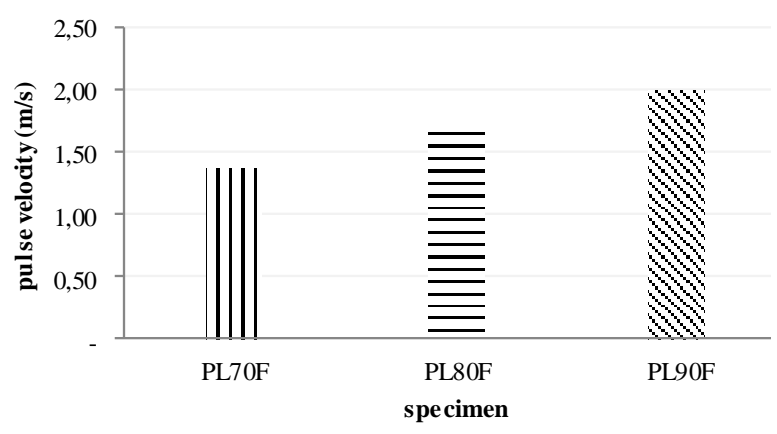

Fig. 4. Ultrasonic Pulse Velocity of Various Core Wall and Fibers

The test results indicated that pulse velocity readings of core thickness of $70 \mathrm{~mm}$ and $80 \mathrm{~mm}$ have similar results, while at core thickness of $90 \mathrm{~cm}$, the results have a difference of $25.96 \%$. The magnitude of this reading difference was due to the alteration of ultrasonic waves by stryrofoam, as well as the effect of the core density values. The waves will be rapidly transmitted from the transducer to the receiver when passing a solid medium, and vice versa [14].

While the result of comparison of pulse velocity value on addition of $1 \%$ fiber for each different thickness of core, indicated that the highest wave reading speed was at the core thickness of $90 \mathrm{~mm}$ with SCM layer thickness equal to $15 \mathrm{~mm}$. This implied that the thicker the plaster is, the more the wave reading will be biased. The refraction of the ultrasonic waves is due to the presence of higher fraction fibers, compared to other compositions. The results of this study were reinforced by Faqih and Priyosulistyo [15] which states that the use of additives as a mixture on concrete, will affect ultrasonic wave readings. Slamet [9] also explains that the use of fiber can cause a snow ball effect, when the composition of the mixture is not homogeneous, so it will cause a large wave refraction difference. The amount of refractive pulse velocity reading for thickness of $20 \mathrm{~mm}$ and $25 \mathrm{~mm}$ is $18 \%$ and $46 \%$ respectively. Therefore, in the application of work evaluation procedures in the field, additive materials (fiber) used for plastering having the thickness of not more than $15 \mathrm{~mm}$, this is to avoid any error in pulse velocity reading process, since it will influence the quality of concrete.

\section{B. Data Normality of the Direct Method}

The statistical data analysis has been proven by Petro and Kim [6], Benaicha et al., [4], Vasanelli, et al., [16], Sabbag and Uyanik [11]. The normal distribution has parameters of the mean and standard deviation. The shape is the infinitely wide coupled bell-shaped curve in both positive and negative directions. A data forms a normal distribution if the amount of data above and below the mean is homogeneous.

The homogeneity test was used to know the composition uniformity of composited wall panel and SCM layers. This test was performed as a prerequisite in the analysis of independent sample t-test and ANOVA. The underlying assumption in the variant (ANOVA) was that the specimen was homogeneous. As the test criterion, if the significance value is more than 0.05 it can be said that the variant of two or more groups of data is homogeneous. 
The data which was analyzed was the value of pulse velocity in the wall panel specimens.

TABLE 5. COV DIRECT METHOD

\begin{tabular}{|c|c|c|c|c|c|c|c|c|c|c|c|c|c|c|c|c|c|c|c|}
\hline \multirow{2}{*}{\multicolumn{2}{|c|}{ Specimen }} & \multicolumn{15}{|c|}{ Measurement points } & \multirow{2}{*}{ mean } & \multirow{2}{*}{ STDEV } & \multirow{2}{*}{$\begin{array}{l}\operatorname{COV} \\
(\%)\end{array}$} \\
\hline & & 1 & 2 & 3 & 4 & 5 & 6 & 7 & 8 & 9 & 10 & 11 & 12 & 13 & 14 & 15 & & & \\
\hline \multirow{2}{*}{ PL70R } & $\mathrm{a}$ & 1.40 & 1.80 & 1.70 & 1.60 & 1.50 & 1.60 & 1.80 & 1.70 & 1.60 & 1.60 & 1.80 & 1.80 & 1.60 & 1.70 & 1.50 & \multirow{2}{*}{1.57} & \multirow{2}{*}{0.15} & \multirow{2}{*}{9.31} \\
\hline & $\mathrm{b}$ & 1.68 & 1.97 & 1.38 & 1.52 & 1.46 & 1.61 & 1.54 & 1.57 & 1.58 & 1.58 & 1.53 & 1.58 & 1.56 & 1.40 & 1.39 & & & \\
\hline \multirow{2}{*}{ PL70F } & a & 1.75 & 1.53 & 1.58 & 1.89 & 1.61 & 1.78 & 1.61 & 1.75 & 1.83 & 1.52 & 1.71 & 1.58 & 1.61 & 1.61 & 1.51 & \multirow{2}{*}{1.91} & \multirow{2}{*}{0.11} & \multirow{2}{*}{5.77} \\
\hline & $\mathrm{b}$ & 2.17 & 2.17 & 2.27 & 2.17 & 2.11 & 2.21 & 2.30 & 2.09 & 2.17 & 1.98 & 1.92 & 2.20 & 2.24 & 2.20 & 2.11 & & & \\
\hline \multirow{2}{*}{ PL80R } & $\mathrm{a}$ & 2.03 & 1.81 & 1.77 & 1.84 & 1.9 & 1.95 & 1.97 & 1.92 & 1.93 & 1.9 & 2.07 & 1.9 & 1.9 & 2.14 & 1.89 & \multirow{2}{*}{2.02} & \multirow{2}{*}{0.13} & \multirow{2}{*}{6.25} \\
\hline & $\mathrm{b}$ & 2.29 & 2.39 & 2.17 & 2.08 & 1.92 & 2.23 & 2.21 & 2.27 & 2.17 & 1.86 & 1.92 & 2.04 & 1.97 & 2.01 & 2.05 & & & \\
\hline \multirow{2}{*}{ PL80F } & $\mathrm{a}$ & 1.75 & 1.9 & 1.73 & 1.85 & 1.71 & 1.78 & 1.78 & 2.01 & 1.80 & 1.80 & 1.83 & 1.79 & 1.89 & 1.98 & 1.84 & \multirow{2}{*}{1.99} & \multirow{2}{*}{0.09} & \multirow{2}{*}{4.70} \\
\hline & $\mathrm{b}$ & 2.17 & 2.17 & 2.27 & 2.17 & 2.11 & 2.21 & 2.3 & 2.09 & 2.17 & 1.98 & 1.92 & 2.2 & 2.24 & 2.20 & 2.11 & & & \\
\hline \multirow{2}{*}{ PL90R } & $\mathrm{a}$ & 1.75 & 1.91 & 1.88 & 1.85 & 1.75 & 1.82 & 1.81 & 1.79 & 1.91 & 1.82 & 1.82 & 1.83 & 1.74 & 1.78 & 1.8 & \multirow{2}{*}{2.02} & \multirow{2}{*}{0.09} & \multirow{2}{*}{4.56} \\
\hline & $\mathrm{b}$ & 2.27 & 2.14 & 2.5 & 2.27 & 2.16 & 2.02 & 2.15 & 2.41 & 2.33 & 2.21 & 2.17 & 2.28 & 2.3 & 2.18 & 2.02 & & & \\
\hline \multirow{2}{*}{ PL90F } & $\mathrm{a}$ & 1.24 & 1.28 & 1.31 & 1.28 & 1.32 & 1.29 & 1.23 & 1.26 & 1.32 & 1.28 & 1.24 & 1.34 & 1.28 & 1.29 & 1.31 & \multirow{2}{*}{1.37} & \multirow{2}{*}{0.13} & \multirow{2}{*}{9.53} \\
\hline & $\mathrm{b}$ & 1.53 & 1.84 & 1.73 & 1.45 & 1.32 & 1.29 & 1.36 & 1.46 & 1.38 & 1.97 & 1.23 & 1.41 & 1.24 & 1.28 & 1.24 & & & \\
\hline
\end{tabular}

The result of variance coefficient analysis shows that in general the use of direct method on the composite wall panel above meets the criteria of homogenous non-destructive test. This has obviously also been proven by Faqih [2], and Yemen, et al. [13]. They state that to determine the criteria of good homogeneity testing, the magnitude of the variance coefficient is not more than $10 \%$. The above data shows that the highest value for the variance coefficient reading is $9.14 \%$ which is below the maximum threshold of $10 \%$.

Furthermore, in the process of data analysis for kolmogorov-smirnov test, the data should be normally distributed as in Table 6.

\section{TABLE 6. KOLMOGOROV SMIRNOV TEST}

\begin{tabular}{|c|c|c|c|}
\hline \multicolumn{2}{|c|}{ Specimen } & Analysis results & Probability \\
\hline PL70R & 0 & $1.43<\mathrm{X}<1.73$ & Normal \\
\hline PL70F & 1 & $1.80<\mathrm{X}<2.18$ & Normal \\
\hline PL80R & 0 & $1.86<\mathrm{X}<2.16$ & Normal \\
\hline PL80F & 1 & $1.55<\mathrm{X}<1.81$ & Normal \\
\hline PL80R & 0 & $1.79<\mathrm{X}<2.25$ & Normal \\
\hline PL80F & 1 & $1.18<\mathrm{X}<1.54$ & Normal \\
\hline
\end{tabular}

The results showed that in general from 180 test points using the direct method, all data was normally distributed. Further, the data can be used for a parametric test.

\section{Homogeneity of Panel Wall}

- One sample Kolmogorov-Smirnov

This test serves to know the data is normally distributed, the details are presented in Table 7.
TABLE 7. ONE SAMPLE KOLMOGOROV-SMIRNOV TEST

\begin{tabular}{|c|c|c|c|c|c|c|c|}
\hline & PL70R & PL70F & PL80R & PL80F & PL90R & PL90F \\
\hline \multicolumn{2}{|c|}{$N$} & 30 & 30 & 30 & 30 & 30 & 30 \\
\hline \multirow{2}{*}{ 逗 } & $\sum_{\Sigma}^{\Xi}$ & $\stackrel{\circ}{\stackrel{n}{n}}$ & כa & $\begin{array}{l}\hat{\sigma} \\
0 \\
i \\
\text { i }\end{array}$ & $\begin{array}{l}\text { ஜn } \\
\infty \\
\text { ô }\end{array}$ & $\begin{array}{l}\text { तิ } \\
\text { రิ } \\
\text { ते }\end{array}$ & $\begin{array}{l}\hat{0} \\
\text { ? }\end{array}$ \\
\hline & $\begin{array}{l}\text { Std. } \\
\text { Deviati } \\
\text { on }\end{array}$ & $\begin{array}{l}\hat{a} \\
\stackrel{n}{0} \\
0\end{array}$ & $\frac{2}{2}$ & $\begin{array}{l}0 \\
\stackrel{0}{0} \\
\text { C. } \\
0\end{array}$ & సิ & 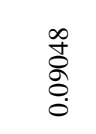 & $\begin{array}{l}\text { t } \\
\text { D } \\
\stackrel{0}{0} \\
0\end{array}$ \\
\hline \multirow{3}{*}{ 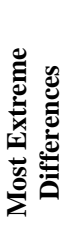 } & $\begin{array}{c}\text { Absolu } \\
\text { te }\end{array}$ & 0.139 & 0.160 & 0.151 & 0.104 & 0.187 & 0.269 \\
\hline & $\begin{array}{c}\text { Positiv } \\
\text { e }\end{array}$ & 0.139 & 0.140 & 0.151 & 0.104 & 0.187 & 0.269 \\
\hline & $\begin{array}{c}\text { Negati } \\
\text { ve }\end{array}$ & -0.101 & -0.160 & -0.075 & -0.081 & -0.128 & -0.225 \\
\hline \multirow{2}{*}{\multicolumn{2}{|c|}{$\begin{array}{c}\text { Test Statistic } \\
\text { Asymp. Sig. } \\
\text { (2-tailed) }\end{array}$}} & 0.139 & 0.160 & 0.151 & 0.104 & 0.187 & 0.269 \\
\hline & & $0.142^{\mathrm{c}}$ & $0.051^{\mathrm{c}}$ & $0.079^{c}$ & $0.200^{\mathrm{c}, \mathrm{d}}$ & $0.087^{\mathrm{c}}$ & $0.069^{\mathrm{c}}$ \\
\hline
\end{tabular}

The test results showed that the overall specimen value of Asymp Sig (2-tailed) on the reference specimen $(0 \mathrm{~kg} / \mathrm{m} 3$ polypropylene fiber). The requirement of a normal distributed sample in this test is greater than $(\alpha>0.05)$, thus the data is normally distributed, then one way anova analysis which is a Parametric Statistics test was performed to find out whether there was an average difference between more than two groups of samples.

\section{- One way Anova}

Anova testing stage was conducted after KolmogorovSmirnov test stated that all specimens were normally distributed. This test served to find out if all specimens of composite panel above wall was homogeneous. Anova test results are presented in Table 8. 
TABLE 8. ONE WAY ANOVA TEST RESULTS

\begin{tabular}{|l|r|r|r|r|r|}
\hline & $\begin{array}{c}\text { Sum of } \\
\text { Squares }\end{array}$ & df & $\begin{array}{c}\text { Mean } \\
\text { Square }\end{array}$ & F & Sig. \\
\hline Between Groups & 11.143 & 5 & 2.229 & 74.027 & .000 \\
Within Groups & 5.239 & 174 & .030 & & \\
Total & 16.382 & 179 & & & \\
\hline
\end{tabular}

Test results in Table 8 shows that on the overall value of Asymp Sig (2-tailed) ( $\alpha>0.05)$. So these six values have the same value of variance. Having the same value of variance, it can be said that the entire sample of Styrofoam wall in this study is homogeneous. Further observations of the difference in mean values of the six groups' variance were analyzed using Post Hoc Test. Given the same variance value, it can be said that all self-compacting mortar data with Styrofoam walls in this study were homogeneous (standard deviation ranging from 0.0448 at the 0.05 significance level).

This means that the Ultrasonic Pulse Velocity Method can be used to find out the homogeneity of the composite panel composite panels with a fairly good measurement accuracy, all composite wall-forming materials composed of well mixed styrofoam, cement, sand and water, as well as the SCM formed as an outer wall layer.

- T-test

T-test serves to test two independent samples. This method was used to determine the effect of using styrofoam, thickness of core composite wall panels and the effect of variation of SCM thickness. Test results are presented in Table 8, 9, and Table 10.

TABLE 8. T-TEST RESULTS CORE PANEL $70 \mathrm{~mm}$

\begin{tabular}{|l|c|c|c|c|c|}
\hline \multirow{2}{*}{$\begin{array}{c}\text { PL70R and } \\
\text { PL70F }\end{array}$} & \multicolumn{2}{|c|}{$\begin{array}{c}\text { Levene's Test for } \\
\text { Equality of } \\
\text { Variances }\end{array}$} & \multicolumn{3}{|c|}{ t-test for Equality of Means } \\
\cline { 2 - 6 } & F & Sig. & $\mathbf{t}$ & $\begin{array}{c}\text { Sig. (2- } \\
\text { tailed) }\end{array}$ & $\begin{array}{c}\text { Std. Error } \\
\text { Difference }\end{array}$ \\
\hline $\begin{array}{l}\text { Equal variances } \\
\text { assumed }\end{array}$ & 11.639 & .001 & -9.302 & .000 & .04200 \\
$\begin{array}{l}\text { Equal variances } \\
\text { not assumed }\end{array}$ & & & -9.302 & .000 & .04200 \\
\hline
\end{tabular}

TABLE 9. T-TEST RESULTS CORE PANEL $80 \mathrm{~mm}$

\begin{tabular}{|c|c|c|c|c|r|}
\hline \multirow{2}{*}{$\begin{array}{c}\text { PL80R and } \\
\text { PL80F }\end{array}$} & \multicolumn{2}{|c|}{$\begin{array}{c}\text { Levene's Test for } \\
\text { Equality of } \\
\text { Variances }\end{array}$} & \multicolumn{3}{|c|}{ t-test for Equality of Means } \\
\cline { 2 - 6 } & F & Sig. & t & $\begin{array}{c}\text { Sig. (2- } \\
\text { tailed) }\end{array}$ & $\begin{array}{c}\text { Std. Error } \\
\text { Difference }\end{array}$ \\
\hline $\begin{array}{l}\text { Equal variances } \\
\text { assumed } \\
\begin{array}{l}\text { Equal variances } \\
\text { not assumed }\end{array}\end{array}$ & 0.926 & 0.340 & 8.868 & 0.000 & 0.03740 \\
\hline
\end{tabular}

TABLE 10. T-TEST RESULTS CORE PANEL 90mm

\begin{tabular}{|c|c|c|c|c|c|}
\hline \multirow{2}{*}{$\begin{array}{c}\text { PL90R and } \\
\text { PL90F }\end{array}$} & \multicolumn{2}{|c|}{$\begin{array}{c}\text { Levene's Test for } \\
\text { Equality of } \\
\text { Variances }\end{array}$} & \multicolumn{3}{|c|}{ t-test for Equality of Means } \\
\cline { 2 - 6 } & $\boldsymbol{F}$ & Sig. & t & \multicolumn{1}{|c|}{$\begin{array}{c}\text { Sig. (2- } \\
\text { tailed) }\end{array}$} & $\begin{array}{c}\text { Std. Error } \\
\text { Difference }\end{array}$ \\
\hline $\begin{array}{l}\text { Equal variances } \\
\text { assumed } \\
\begin{array}{l}\text { Equal variances } \\
\text { not assumed }\end{array}\end{array}$ & 7.734 & 0.007 & 12.264 & 0.000 & 0.05346 \\
\hline
\end{tabular}

Based on the independent t-test results it can be stated that the wall panel using polypropylene fiber of $1 \mathrm{~kg} / \mathrm{m}^{3}$ affects the workability level. The more fiber, the more difficult the workability becomes (slump flow value / filling ability) becomes smaller. These results are similar to those studied by Agus et al. [1],, Slamet [9], and reinforced by the results of statistical analysis in this study which states that in Table 7; Table 8 and Table 9, the Sig level (2-tailed) $0.00<0.05$ which means that in the presence of polypropylene fiber is very influential with workability.

\section{The Accuration of the Direct Method Reading.}

Constraints in the test results were the refraction of waves by Styrofoam material and polypropylene fiber. Therefore, it is necessary to analyze the accuracy of direct method readings in Fig. 5 and Fig. 6.

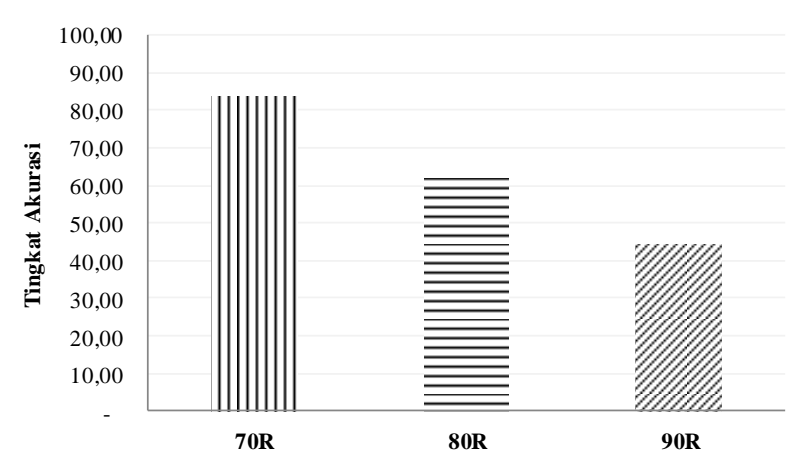

Fig. 5. Accuracy of Pulse Velocity Readings of Variations of Styrofoam Cores

Note:

70R: Core thickness of $70 \mathrm{~mm}$, Reference

Based on Fig. 5, the reading accuracy level will be very good when the thickness of the core is smaller. The magnitude of the difference in the value of $70 \mathrm{~mm}$ core accuracy rate compared to $80 \mathrm{~mm}$ and $90 \mathrm{~mm}$ is $35 \%$ and $90 \%$. The thicker the core of the Styrofoam wall panels are, the more biased the readings will be. This is because the core panel contains a more dominant styrofoam composition, pulse velocity will flow well when passing solid media [17], but it will be biased as it passes through hollow media. As comparison. It is presented Fig. 6.

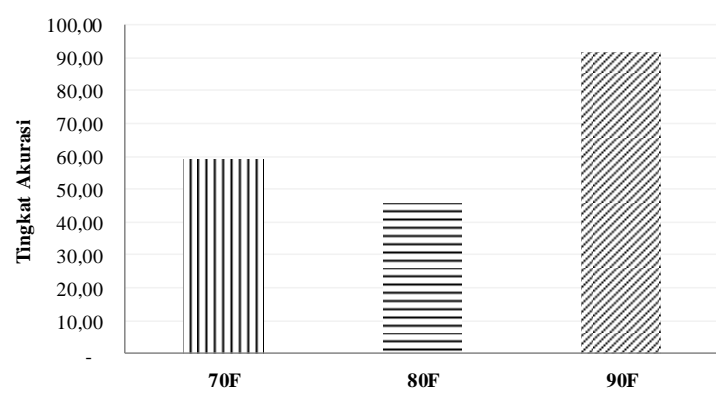

Fig. 6. The Accuracy of the Direct Method

Note:

70F: Core thickness of $70 \mathrm{~mm}$, using SCM with $1 \mathrm{~kg} / \mathrm{m}^{3}$ Polypropylene Fiber 
Fig. 6 shows that the best pulse velocity reading at $90 \mathrm{~mm}$ core thickness with SCM is $15 \mathrm{~mm}$. The effect of polypropylene fiber in refracting waves is quite large. When compared with the pulse velocity core Styrofoam readings, the difference is $41 \%$. The worst pulse velocity readings is at $70 \mathrm{~mm}$ core thickness (inversely proportional to Fig. 5). In general, the direct method has very good accuracy, as reported by Budi [18] which states that the results of the direct method testing aims to find out the compressive strength of the concrete [19] and concrete homogeneity test, because it has a very high accuracy. The accuracy is $9 \%$ and 5\% higher than the indirect and semi direct methods Turgut and Kucuk [20]. The results of this test means that SCM thickness is very influential on pulse velocity reading, it also proves the validity of statistical test compared with the results of laboratory experiments.

\section{CONCLUSION}

Non-destructive test methods using UPV have been used to evaluate the characteristics of lightweight wall panels. Based on the comprehensive test, the following conclusions are taken:

- The use of Styrofoam as the main material of composite wall panels is very useful for reducing environmental pollution.

- Polypropylene fibers are materials that can refract ultrasonic waves, however in the case of homogeneity testing, the effect of wave refraction is not very large, because the reliability of the direct method so as to show excellent results.

- Statistical analysis and direct test method results to determine the homogeneity of composite wall panels showed satisfactory results with a $90 \%$ accuracy.

\section{ACKNOWLEDGMENT}

The Author wish to thank Faculty of Engineering, Universitas Negeri Yogyakarta for financial support and supplying the materials used in the experiments.

\section{REFERENCES}

[1] Agus Santoso, Slamet Widodo, and Faqih Ma'arif, "Pemanfaatan Limbah Styrofoam (Expanded Polysterene) Untuk Pembuatan Dinding Struktural Beton Ringan Ramah Lingkungan”, INERSIA: , 2011, Vol. 07, May No.1. 1-18.

[2] Faqih, Ma'arif, Priyosulistyo, Ashar Saputra, "The effect of variation of axial column and moment under cyclic loading", Gadjah Mada University, 2010, xxiii, 181 p., bibl., ills., $29 \mathrm{~cm}$.
[3] Benaicha, Mouchine et.al., "Correlation between the mechanical behavior and the ultrasonic velocity of fiber-reinforced concrete". Elsevier: Construction and Building Materials 101, 2015, 702-709

[4] Benaicha, M. et al., "Prediction of self-compacting concrete homogeneity by ultrasonic velocity". Elsevier: Alexandria Engineering Journal, 2015, 54, 1181-1191.

[5] Al-Mufti, R. Latif and Fried, A.N., "Pulse velocity assessment of early age creep of concrete".Elsevier: Construction and Building Materials $121,2016,622-628$

[6] Petro Jr, John T and Kim, Jubum, "Detection of delamination in concrete using ultrasonic pulse velocity test". Elsevier: Construction and building materials, 26, 2012, 574-582.

[7] M. Hamidian, M. Shariati, M.M.K. Arabnejad, H. Sinaei, "Assessment of high strength and light weight aggregate concrete properties using ultrasonic pulse velocity technique", Int. J. Phys. Sci. 6 (22) (2011) 5261-5266.

[8] Lorenzi, Alexandre, et.,al, "Ultrasonic pulse velocity in concrete specimens". AAENDE: IV Confrencia Panamericana de END Buenos Aires-October, 2017.

[9] Slamet, Widodo, "Fresh and hardened properties of Polypropylene fiber added Self-Consolidating Concrete", International journal of civil and structural engineering, ISSN 0976 - 4399, 2012, Volume 3, No 1.

[10] V.M. Malhotra, N.J. Carino (Eds.), Handbook on Non Destructive Testing of Concrete, CRC Press LLC, 1991.

[11] Sabbag, Nevbahar, and Uyanik, Osman, "Prediction of concrete strength by ultrasonic velocities". Elsevier: Journal of Applied Geophysics, 141, 2017, 13-23.

[12] Zoidis, Nikolaos, et.al., "Inspection, evaluation and repair monitoring of cracked concrete floor using NDT methods", Elsevier: Construction and building materials, Article in press.

[13] Yaman, Ozgur, Ismail, "Ultrasonic pulse velocity in concrete using direct and indirect transmission". ACI Materials Journal, NovemberDecember 2001, Title no. 98-M48.

[14] Pundit, Pundit Manual for use with the Portable Ultrasonic NonDestructive Digital Indicating Tester, C.N.S. Electronics LTD, 1991.

[15] Faqih, Ma'arif and Priyosulistyo, "The characteristic of ultrasonic pulse velocity (UPV) on mortar with polypropylene fibers as additives," 1st SCESCM, Universitas Gadjah Mada, Yogyakarta.

[16] Vasanelli, Emilia, "Ultrasonic pulse velocity for the evaluation of physical and mechanical properties of a highly porous building limestone". Elsevier: Ultrasonics, 2015, February.

[17] Sujiani, Jepriati, and Proyosulistyo, "Accuracy test of Ultrasonic Pulse Velocity (UPV) for detecting the depth of Cracks on concrete". Gadjah Mada University, 2008, xvi, 101 p., bibl., ills., $29 \mathrm{~cm}$.

[18] Budi, N., and Priyosulistyo, "Ultrasonic wave velocity relationship on different variations of concrete quality using local materials. Gadjah Mada University, xvii, 118 p., bibl., ills., $29 \mathrm{~cm}$.

[19] Hannachi, Samia and Guetteche, M.N., "Review of the ultrasonic pulse velocity Evaluating concrete compressive strength on site". Scientific cooperations international workshops on engineering branches 8-9 August, 2014, Koc. University, Instanbul, Turkey.

[20] Turgut, P and Kucuk, F, "Comparative relationships of direct, indirect, and semi-direct ultrasonic pulse velocity measurements in Concrete", ISSN 1061-8309, Russian Journal of Nondestructive Testing, 2006, Vol. 42, No. 11, pp. 745-751. @) Pleiades Publishing, Inc., 2006. 